\title{
Medialität der Metrik: Eine Königsberger Bildungskontroverse um 1580 im Spiegel der Kasualdichtung
}

\author{
Regina Toepfer
}

Für die Ablösung mittelhochdeutscher Versepen durch frühneuhochdeutsche Prosaromane werden in der Literaturgeschichte sprachhistorische, soziologische, rezeptionsästhetische und inhaltlich-legitimatorische Gründe angeführt. ${ }^{1}$ Wie ist jedoch ein gegenteiliges Phänomen zu erklären, wenn ein Autor Ende des sechzehnten Jahrhunderts einen in Prosa verfassten griechischen Text in lateinisches Versmaß übersetzt? Diese Frage nach dem „Warum“ einer Versifizierung stellt sich, wenn man die Drucküberlieferung des Kirchenvaters Basilius von Cäsarea betrachtet. ${ }^{2}$

Basilius, der schon zu seinen Lebzeiten den Beinamen „der Große“ erhielt, gehört zu den bedeutendsten Autoren, die von den Humanisten neu entdeckt und dem lateinischen Abendland erschlossen werden. ${ }^{3}$ Seine rhetorische Begabung und seine thematische Vielseitigkeit, die Anknüpfungspunkte für Humanisten, Pädagogen und Kontroverstheologen bietet, sorgen für die große Beliebtheit des griechischen Kirchenvaters. Allein im deutschen Sprachraum erschienen im fünfzehnten und sechzehnten Jahrhundert mindestens 125 Drucke seiner Schriften; darunter befinden sich mehrere Gesamt- und Teilausgaben, aber auch Sammelbände, Schulbücher und zahlreiche Vorlesungshefte. ${ }^{4}$ Die Rezeption seiner vielgerühmten Werke verläuft dabei meist über die Vermittlungsstufe des Lateinischen, nur ein geringer Teil wird im griechischen Original

1 Zum Übergang vom Reimvers zur Prosa vgl. Schnell (1984) 214-248; Besch (1972); Brandstetter (1971); Henne (1978).

2 Diese habe ich im Kontext meiner Dissertation systematisch erschlossen und historisch kontextualisiert. Vgl. Toepfer (2007).

3 Vgl. auch Backus (1990); Schucan (1973).

4 Vgl. die Druckdokumentation in Toepfer (2007) 449-617. 
oder in einer deutschen Übertragung publiziert. ${ }^{5}$ Die über neunzig lateinischen Druckausgaben orientieren sich fast alle an der von Basilius verwendeten Form der Prosa; um so bemerkenswerter ist der eine rezeptionsgeschichtliche Sonderfall: Im Jahr 1580 erscheint bei Georg Osterberger in Königsberg eine Predigt des Kirchenvaters, die in gebundene Sprache überführt worden ist. ${ }^{6}$

Auf dem Titelblatt des Drucks werden nicht nur der Ausgangstext und sein Verfasser, sondern auch die Art der Bearbeitung und der Verantwortliche für diesen Prozess vorgestellt (vgl. Abb. 1). Zugrunde liegt die ORATIO DIVI BASILII DE NOCENTISSIMo INVIDIAE VICIO, die Rede des Heiligen Basilius über das äußerst verwerfliche Laster des Neids, die von einem Übersetzer namens Josua Thomae CARMINE HEROICO TRANslata et illustrata, als episches Gedicht übertragen und erläutert wurde. Schon durch die optische Hervorhebung seines Namens, vor allem aber durch seine Bezeichnung als Autor wird der große Anteil Thomaes an der Gestaltung der Ausgabe zum Ausdruck gebracht. In der unteren Mitte des Titelblatts zentral positioniert, sticht der Name des Übersetzers aufgrund seiner isolierten Stellung ins Auge. Dass Josua Thomae auf dem Titelblatt zum Ko-Autor erklärt wird, ist für die humanistische Antikenrezeption sehr ungewöhnlich. Obwohl viele Gelehrte ihre sprachlichen Fähigkeiten und editorischen Leistungen herausstellen, bleibt ihr Bildungsstolz doch stets ein abgeleiteter und auf die geschätzten Autoritäten der Antike bezogen. ${ }^{7}$ Thomae hingegen wird, vermutlich weil er bei seiner Übersetzung die antike Vorlage formal vollkommen neu gestaltet und sie auch inhaltlich ergänzt hat, sogar ein Autorenstatus zugestanden. Welche Motive könnten den Interpreten veranlasst haben, die antike Oratio in ein frühneuzeitliches Carmen zu verwandeln? Eine Antwort auf diese Frage dürfte in dem Bereich der Medialität zu suchen sein, wie im Folgenden gezeigt werden soll. ${ }^{8}$ Mit der metrischen Übertragung erhält der Text einen spezifischen medialen Status, so meine These, der bestimmten Rezeptionsbedingungen geschuldet ist und eine eigene Vermittlungssituation evoziert. Erste Aufschlüsse über die Zweckbestimmung des

5 Vgl. Toepfer (2007) 19.

6 Vgl. vD 16, B 716, und Toepfer (2007) 536-538 (= Inv 5).

7 Vgl. auch Kleinschmidt (1997); Müller (1995); Toepfer (2007) 136-144. Bemerkenswert ist auch die fehlende Angabe einer Vorlage, so dass nicht einmal deutlich wird, ob Thomae auf der Grundlage einer griechischen Edition eine eigene Version anfertigt oder ob er sich auf eine lateinische Vorlage stützt und diese entsprechend verändert. Zu dem Zeitpunkt seiner Übersetzung sind im deutschen Sprachraum sowohl zwei griechische als auch sieben lateinische Gesamtausgaben des Basilius publiziert worden, die Thomae grundsätzlich zur Verfügung stehen könnten (zu den Druckausgaben vgl. Toepfer (2007) 555-580).

8 Grundlegend zur historischen Medialität vgl. Kiening (2007) und Kellermann (2004). 


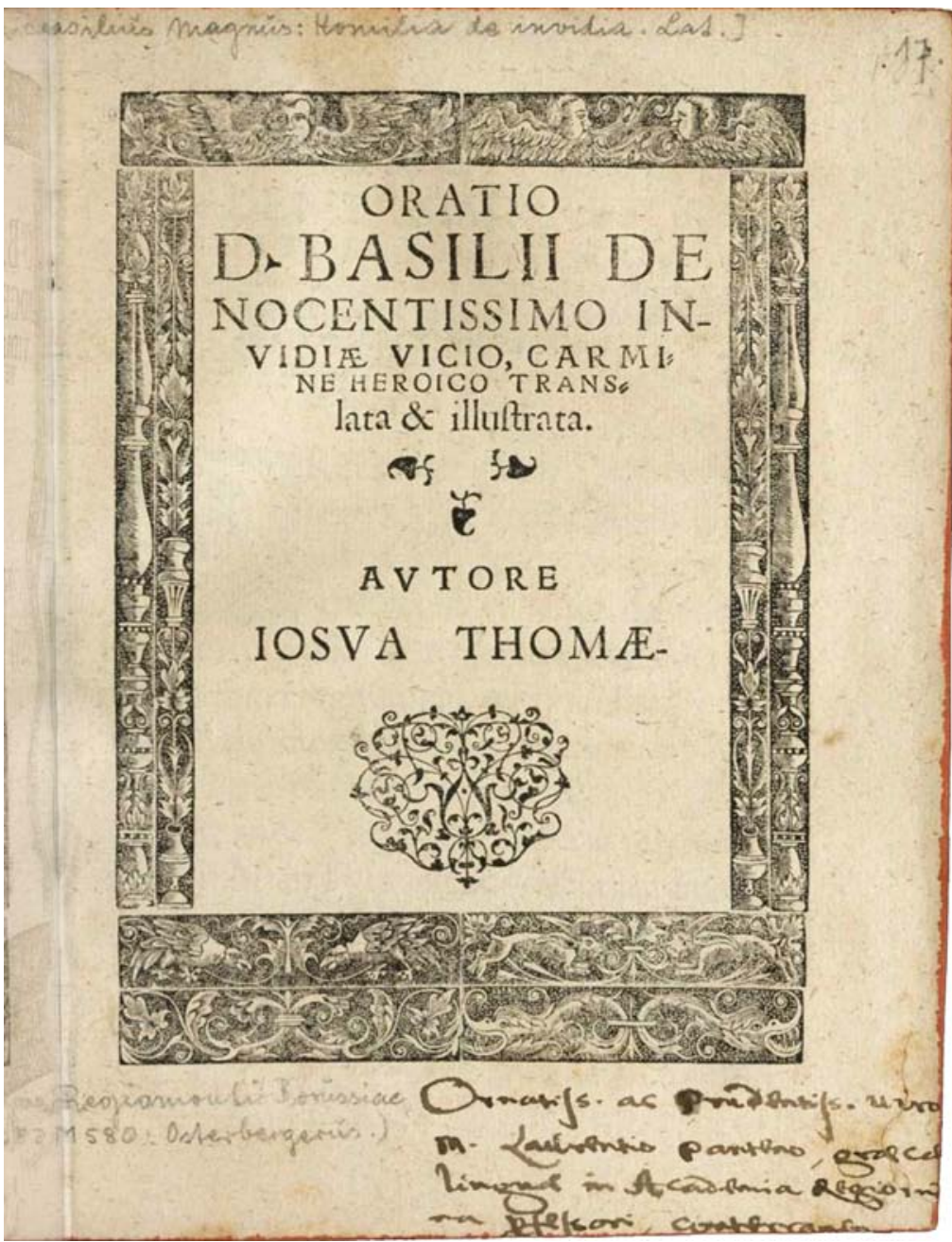

AвB. 9.1 Basilius [Caesariensis/ Magnus], Oratio De Nocentissimo Invidiae Vicio [= Homilia de invidia], Carmine Heroico Translata \& illustrata, Autore Iosua Thomae, Osterberger: Königsberg 1580, Titelblatt VERWENDETES EXEMPLAR: WOLFENBÜTTEL, HERZOG AUGUST BibliotheK, A: 49.1 POET. (17), DigitalisieRt ALS HTTP://Diglib .HAB.DE/DRUCKE/49-1-POET-17S/00001.JPG, AUFGERUFEN AM 1. FEBRUAR 2018 
Königsberger Drucks sind von dem Paratext zu erhoffen, ${ }^{9}$ den Thomae für seine Übersetzung verfasst und als Beigabe zur Veröffentlichung bestimmt hat.

\section{Das Widmungsschreiben Josua Thomaes: Ein Plädoyer für die Bildung}

Wie bei humanistischen Drucken üblich, ${ }^{10}$ leitet Josua Thomae seine Textausgabe mit einem Widmungsbrief ein, mit dem er sich an konkrete Adressaten wendet und ihnen seine Version zueignet: „AMPLISSIMIS, PRVDENTISSIMIS, ATQVE ORNATISSImis ETC. TRIVM Ciuitatum Regijmontis Borussiae, Veteris, Kniphouianae et Libenicensis, Consulibus, Patribus et Scabinis, Patronis ac Dominis suis omni obseruantia colendis. ${ }^{111}$ Sein Schreiben an die auf diese Weise gerühmten Räte der drei Städte Königsberg, nämlich Altstadt, Kneiphof und Löbenicht, beginnt mit allgemeinen Ratschlägen für eine gute Regierung. Mit dem Verweis auf eine biblische Autorität, Salomon, und auf den antiken griechischen Geschichtsschreiber Xenophon, erklärt Thomae, wie ein Reich und ein Volk am besten gelenkt werden sollten. Während Gewalt und Tyrannis nur Hass, Spaltungen und Unfrieden erzeugten, werde eine Herrschaft durch Eintracht, Ruhe und Freundschaft gestützt. Daher sollten eher Wohltaten verteilt und Freunde gewonnen werden, statt Untergebene das Fürchten zu lehren, sich viele Feinde zu verschaffen und zugleich Neid zu schüren. ${ }^{12}$

In einem nächsten Schritt konkretisiert Thomae diese allgemeinen Empfehlungen und Warnungen, indem er sich auf die Predigt des Kirchenvaters bezieht und den Neid in den Mittelpunkt seiner Betrachtung rückt. Vor diesem schrecklichen und gefährlichen Laster müsse man sich besonders in Acht nehmen, wie viele Gelehrte zurecht betont hätten. Dass er sich unter den vielen Mahnreden ausgerechnet für die des Basilius entschieden habe, begründet Thomae mit der doppelten Zielrichtung der Predigt De invidia. Basilius beschreibe nicht nur die Beschaffenheit und Größe, die Ursache und die Wirkungen dieses Lasters, sondern zeige auch prophylaktische und therapeutische

$9 \quad$ Grundlegend zu diesem Begriff vgl. Genette (2001).

10 Vgl. z. B. Leiner (1965); Schwitzgebel (1996); Toepfer (2008/2009); Vogel (1999).

11 Thomae (1580) [VD 16, B 716, Exemplar der Herzog August Bibliothek Wolfenbüttel, A: 49.1 Poet. (17) unter http://diglib.hab.de/drucke/49-1-poet-17s/start.htm am 17. April 2013], Sign. A2a. Übers.: „An die bedeutendsten, klügsten und ehrenvollsten etc. Bürgermeister, Ratsherrn und Schöffen der drei Städte Königsberg, Altstadt, Kneiphof und Löbenicht, seine mit aller Hochachtung zu verehrenden Gönner und Herren." - Die Abbreviaturen sind hier wie im Folgenden aufgelöst. 
Mittel auf. ${ }^{13}$ Den Gewinn, den sich der Übersetzer von dieser Rede erhofft, macht er bemerkenswerter Weise von einer metrischen Form abhängig: „Hanc Diui Basilij orationem, dum euoluerem, visum est, non abs re fore, si aliquando carminis quodam genere illustraretur. ${ }^{14}$ Auch wenn Thomae nicht näher darauf eingeht, weshalb er ein Carmen als geeignetes Medium ansieht, muss der Gattungstransfer doch als konstitutiv für seine Übersetzungsmotivation angesehen werden.

Der Brief schließt mit einer Hinwendung zu den Adressaten, denen Thomae sein Werk übereignet, und einer Begründung dieser Widmung. Seine Empfänger werden als bedeutendste, klügste, ehrenwerteste und bei weitem gebildetste Männer gewürdigt, die sich durch ihr Interesse und ihren Einsatz für die studia bonarum artium auszeichneten. ${ }^{15}$ Dass eine solche Haltung in Königsberg nicht unumstritten ist, geht aus der Fortsetzung der Argumentation hervor. Sein Geschenk will Thomae als Zeichen seiner Hochachtung für die Ratsherrn verstanden wissen, wohingegen er sich „contra Theoninos dentes“, gegenüber bösen Zungen, abgrenzt. Welche Gegner er mit dieser horazischen Wendung ${ }^{16}$ zu treffen sucht, ist auch dem Schlussappell nicht eindeutig zu entnehmen, bei dem der Briefsteller zwei konkrete Bitten an die Ratsangehörigen formuliert: Zum Ersten werden sie aufgefordert, die Verleumdungen der Neider zurückweisen, und zum Zweiten gebeten, ihre positive Einstellung gegenüber den Freunden der Musen beizubehalten. ${ }^{17}$ Andernfalls, dessen ist sich Tho-

13 Vgl. Thomae (1580) Sign. A3a: „Inter quos (meo iudicio) vel principem locum Diuus obtinet Basilius. Qui $\varphi v \sigma 10 \lambda \circ \gamma(x \eta \dot{v} v$, seu magnitudinem huius vicij, monstrata causa et positis

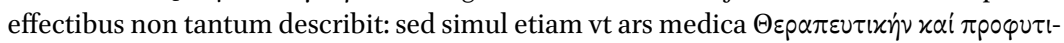

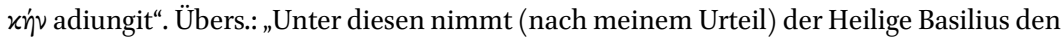
ersten Platz ein. Der die natürliche Beschaffenheit oder die Größe dieses Lasters durch die aufgezeigte Ursache und die dargelegten Wirkungen nicht nur beschreibt, sondern ebenso auch wie die Heilkunst etwas Therapeutisches und Vorbeugendes hinzufügt."

14 Thomae (1580) Sign. A3a. Übers.: „Diese Rede des Heiligen Basilius schien, während ich sie las, nicht ohne Nutzen zu sein, wenn sie einmal in der spezifischen Gattung des Gedichts erhellt würde."

15 Thomae (1580) Sign. A3b: „Quicquid autem istuc est, vobis Amplissimis Prudentissimis et Ornatissimis etc. Patribus, quibus bonarum artium studia curae et cordi adhuc esse quilibet testatur, dedicatum esse volui“. Übers.: „Alles was hingegen dieses betrifft, wollte ich, dass es euch, bedeutendste, klügste und ehrenvollste etc. Ratsherrn, gewidmet ist, denen ein jeder bezeugt, dass bei euch die Studien der guten Künste Unterstützung finden und sie euch so sehr am Herzen liegen.“

16 Vgl. die Epistulae in der Ausgabe Fink (2003) lib. 1, 18, 82.

17 Thomae (1580) Sign. A3b: „Interea vos Patres longe humanissimi, rude ac leuidense hoc meum munus aequo animo suscipite, boni consulite, hoc qualecunque, meae erga vos obseruantiae symbolum contra Theoninos dentes, et obtrectatorum calumnias defendite, et musarum cultoribus, vt facitis, fauete: [...]“. Übers.: „Nehmt ihr einstweilen, bei weitem 
mae gewiss, würden die Schulen und Studien in Königsberg ebenso zugrunde gehen, wie sie in Kleinasien und in zahlreichen Orten Europas bereits verfallen seien. ${ }^{18} \mathrm{Zu}$ denken ist bei diesem Hinweis auf die missliche Bildungssituation sowohl an die militärische Bedrohung durch die Türken im Osten als auch an die Krise der deutschen Universitäten in der Mitte des sechzehnten Jahrhunderts, die in engem Zusammenhang mit den religiösen und konfessionellen Auseinandersetzungen steht. ${ }^{19}$ Diese Gefahren vor Augen warnt Thomae die Ratsherren vor einer Vernachlässigung der Bildung in ihrer Stadt. Der Schaden für Kirche und Gemeinwesen wäre, wie die Beispiele anderer Orte zeigen sollen, beträchtlich.

\section{Historischer Kontext: Auseinandersetzungen um die Albertina}

Thomaes Widmungsbrief zu seinem Carmen heroicum lässt auf eine lokale bildungspolitische Kontroverse schließen, die um das Jahr 1580 in der Stadt am Pregel virulent gewesen sein muss. In der umfangreichen Forschungsliteratur zur Geschichte der Stadt und der Universität Königsberg wird das Reformationsjahrhundert insgesamt als ein sehr bedeutendes, aber zugleich unruhiges und zerrissenes Zeitalter dargestellt. ${ }^{20}$ Erst nachdem das frühere Deutschordenland in einen protestantischen Territorialstaat verwandelt worden war und Herzog Albrecht von Preußen diesen von dem polnischen König als erbliches Lehen empfangen hatte, begann Königsberg bildungsgeschichtlich eine nennenswerte Rolle zu spielen. ${ }^{21}$ Der neue politische und kirchliche Verwaltungsapparat sorgte für einen steigenden Bedarf an Beamten, so dass sich der

gebildetste Herren, dieses mein ungeschliffenes und geringfügiges Werk mit gnädigem Herzen an, heißt es gut, verteidigt dieses wie auch immer beschaffene Zeichen meiner Hochachtung gegenüber euch gegen böse Zungen und die Verleumdungen der Neider und seid den Freunden der Musen, wie ihr es tut, gewogen [...].“

18 Thomae (1580) Sign. A3b: „[...] ne studia quoque et Scholae apud vos, quemadmodum proh dolor in Asia et plurimis Europae locis, magno cum Ecclesiae et Reipublicae detrimento fieri videmus, in praeceps eant“. Übers.: „[...] damit die Studien und die Schulen bei euch nicht auch, wie wir sehen, dass es, oh Schmerz, in Kleinasien und in den meisten Orten Europas, zum großen Schaden von Kirche und Staat geschieht, jäh zugrunde gehen."

19 Zur deutschen Universitätskrise vgl. Seifert (1984) 145, und Asche (2001).

20 Zu der problematischen Quellensituation nach der Zerstörung Königsbergs 1944-1945 und der Forschungsgeschichte, ausgehend vom siebzehnten Jahrhundert bis zur Gegenwart vgl. Komorowski (2008); Walter (2004; 2005); Jähnig (2001); Lawrynowicz (1999) und Von Selle (1956).

21 Von einem "nahezu vollkommenen Neubeginn“ spricht in diesem Zusammenhang Daugsch (1994) 9 . 
Landesherr selbst um eine angemessene Ausbildung seiner Untertanen kümmerte. Nach ersten Ansätzen einer Schulreform erfolgte 1544 die Gründung der Universität Königsberg, der Albertina, wie sie in Anlehnung an ihren Stifter benannt wurde. ${ }^{22}$ Mit ihren Statuten orientierte sich die nach Marburg zweite, neugegründete protestantische Landesuniversität an dem Vorbild Wittenbergs. ${ }^{23}$ Obwohl Herzog Albrecht bei seinen Plänen Unterstützung von prominenten Reformatoren erhielt und er Melanchthons Schwiegersohn Georg Sabinius als ersten Rektor gewinnen konnte, gelang es ihm nicht, die Universität in gewünschter Weise zu stabilisieren. ${ }^{24}$ Namhafte Gelehrte wie Joachim Camerarius verzichteten dankend auf ihre Berufung in die preußische Provinz. ${ }^{25}$ Manche Lehrstühle konnten in Ermangelung geeigneter Interessenten über längere Zeit nicht besetzt werden. Die nominierten Königsberger Professoren hingegen lieferten sich erbitterte Kämpfe um die theologische Wahrheitsfindung, wie der berühmt-berüchtigte Streit um Andreas Osiander zeigt. ${ }^{26}$ Vor allem aber sorgten eine unzureichende Besoldung und die ungleiche Ausstattung von Lehrstühlen für Ärger und Proteste. ${ }^{27}$

Nachdem Herzog Albrecht im Jahr 1568 gestorben war, verschärften sich die politischen Auseinandersetzungen um die Universität und ihre Finanzierung: ${ }^{28}$ Seit Ende der sechziger Jahre beklagten sich die Professoren, mit den geringen Gehältern nicht auskommen zu können. Die vom Herzog eingesetzten Regimentsräte machten sich diese Beschwerde zu eigen und legten im preußischen Landtag einen Antrag zur besseren Besoldung der Professoren vor, ohne jedoch bei den Ständen auf Zustimmung zu stoßen. ${ }^{29}$ Vielmehr zeigten sich der erste und der zweite Stand ihrerseits mit dem Verhalten der Universitätsbediensteten unzufrieden und stellten gar die Existenz der Bildungsinstitution als solcher in Frage. So fühlte sich der zweite Stand, bestehend aus Ritterschaft und Adel, durch die Gerichtsdienste der Professoren in seinen privaten Interessen benachteiligt. Statt zu viele außeruniversitäre Tätigkeiten wahrzunehmen, sollten sich die Lehrenden der juristischen Fakultät besser um ihre Kollegien kümmern.

\footnotetext{
22 Zur Gründung vgl. v. a. Lawrynowicz (1999) 38-50; Walter (2005) 23-24.

23 Vgl. Daugsch (1994) 13, 15; Lawrynowicz (1999) 61-62; Moeller (1995).

24 Vgl. Komorowski (2008) 1-2; Von Selle (1956) 9-17 und Mundt (2008).

25 Vgl. Von Selle (1956) 10-11, 27-28.

26 Vgl. Von Selle (1956) 36-54; Lawrynowicz (1999) 63-69; Moeller (2001) 21-22; Dembowski (2001) 25-27.

27 Vgl. Daugsch (1994) 18; Von Selle (1956) 17-18; Moeller (2001) 22.

28 Vgl. Töppen (1849) 571-572, und Von Selle (1956) 68-69.

29 Zu den Entscheidungsprozessen und der Zusammensetzung des preußischen Landtages vgl. Körber (1998) 53-85, 98-120.
} 
Noch weitreichender war die Kritik des ersten Standes. Die Herren und Landräte vertraten die Ansicht, dass Rektor und Senat ihre Privilegien missbrauchten und die Professoren mehr auf Einladungen aus seien, als Vorlesungen zu halten. ${ }^{30}$ Diese Vorhaltungen gipfelten in dem Vorwurf, dass Landeskinder an fremden Hochschulen besser ausgebildet würden als an der eigenen, womit die Universität Königsberg ihren eigentlichen Zweck nicht mehr erfülle. Wenn sich diese defizitäre Situation nicht ändere, argumentierte der erste Stand, sollten die Stiftungsgelder für andere Ziele eingesetzt werden. Nur der dritte Stand, die Vertreter der Städte, verteidigte die Universität und forderte, die Professoren angesichts solch massiver Beschuldigungen selbst anzuhören. Obwohl sich die Städte dafür aussprachen, die Gehälter zu erhöhen, scheiterte die Initiative der Regimentsräte aufgrund der massiven Widerstände des ersten und zweiten Stands.

DerWidmungsbrief Josuae Thomaes spiegelt somit die konfliktreichen Umstände der Zeit, in der die Übersetzung der Predigt De invidia des Kirchenvaters entstanden ist. Thomae, der am 27. November 1579 an der Albertina immatrikuliert worden ist und dort am 5 . April 1582 zum Magister promoviert werden wird, ${ }^{31}$ bezieht mit seinem Text 1580 Stellung in der bildungspolitischen Kontroverse, die zwischen den Universitätsangehörigen und den Ständen geführt wird. Sein Lob für die Ratsherrn der drei Städte Königsberg ist angesichts dieser Debatte allzu verständlich: Ihr Einsatz für eine bessere finanzielle Ausstattung der Universität macht sie zu Freunden und Förderern der Musen, der Studien und der Wissenschaft. Der nebulöse Aufruf Thomaes, die Verleumdungen der Neider zurückzuweisen, dürfte sich hingegen auf die Kritik der Vertreter des ersten und zweiten Standes beziehen. Deren Vorwürfe gegenüber den Professoren, ihre akademischen Pflichten nicht zu erfüllen, werden als haltlose Unterstellungen entlarvt, die aus Neid an der Förderung der Universität erwachsen sind. Mit der Wahl des übersetzten Werks verfolgt Thomae die Strategie, eine allgemein anerkannte Autorität an der Stelle der verunglimpften Universitätsangehörigen sprechen zu lassen. ${ }^{32}$ Die Worte des Kirchenvaters sollen die Bildungsgegner mit ihrem Fehlverhalten konfrontieren, sie eines Besseren belehren oder sie zumindest als unchristlich bloßstellen.

Wie prekär die Lage der Albertina ist, wenn sie keine finanzielle Unterstützung von den Königsberger Ratsherrn erhält, wird in den jüngsten politischen

$30 \quad$ Zu den Privilegien vgl. Daugsch (1994) 16-17.

31 Vgl. Erler (1910-1917) Bd. 1, 70; Komorowski (1988) Nr. 328.

32 Zur allgemeinen Beliebtheit dieses Verfahrens bei den humanistischen Gelehrten vgl. Toepfer (2007) 273-287, 412-420. 
Ereignissen unmittelbar vor der Veröffentlichung des Drucks offensichtlich. ${ }^{33}$ Kurzfristig verbessert sich die finanzielle Situation der Hochschule, nachdem Markgraf Georg Friedrich von Brandenburg 1578 die Vormundschaft für den gemütskranken Erben des Landes, Herzog Albrecht Friedrich, übernommen hat. Der Markgraf erhöht die Gehälter der Professoren, er besetzt freie Lehrstühle und gewinnt einige erfolgreiche und engagierte Dozenten, zu denen unter anderem der Gräzist Laurentius Pantenus zählt. ${ }^{34}$ Nur ein Jahr später versiegt jedoch die wichtigste Finanzierungsquelle des Landes, so dass die Maßnahmen zugunsten der Universität kaum länger durchführbar sind. Die Vertreter der Städte Königsberg weigern sich, die von der Regierung geforderten Zahlungen zu entrichten, und nutzen so ihre Einflussmöglichkeit, im preußischen Landtag selbst Politik zu gestalten. Weil die Städte den größten Anteil an Steuern aufbringen, lässt die fehlende Zahlungsbereitschaft Königsbergs 1579/80 das gesamte Steuersystem des Herzogtums zusammenbrechen. ${ }^{35}$ Auch wenn sich die Opposition der Städtevertreter Königsbergs nicht gegen die Albertina und ihre Professoren richtet, muss sich ihr Verhalten dennoch sekundär auf die lokale Bildungseinrichtung auswirken und diese in ihrer Existenz bedrohen.

Thomaes Appell an die Ratsherrn der Städte Königsberg gewinnt vor diesem Hintergrund an politischer Aktualität und Brisanz. Ohne die Steuerbewilligung der Städte kann die einzige Universität des Landes nicht länger finanziert werden, wodurch die Studien der guten Wissenschaften in Königsberg und damit in ganz Preußen tatsächlich zugrunde gingen. Daher ist es für Thomaes Argumentation von zentraler Relevanz, an die bisherige positive Haltung der Räte Königsbergs gegenüber ihrer Universität zu erinnern und um eine Fortsetzung dieser Förderung zu bitten. Nur wenn die Städte finanzielle Mittel bereitstellen, kann der Hochschulbetrieb aufrechterhalten werden. Statt diesen Zusammenhang näher auszuführen, lenkt Thomae in seinem Widmungsschreiben sowie mit der Wahl seines Textes Über den Neid die Aufmerksamkeit auf die Widersacher der Bildungsbewegung. Durch die Abgrenzung von den missgünstigen Gegnern, die im ersten und zweiten Stand zu verorten sind, konstituiert Tho-

33 Seltsamerweise findet sich in der Literatur zur Königsberger Universitätsgeschichte kein Hinweis auf die Auseinandersetzungen im preußischen Landtag Ende der siebziger Jahre des sechzehnten Jahrhunderts.

Zu der Einstellung neuer Lehrender vgl. Von Selle (1956) 7o. Der aus Rügenwalde in Pommern stammende Laurentius Pantenus (Pantaenus) wurde am 27.03.1572 an der philosophischen Fakultät der Albertina zum Magister promoviert. Vgl. Komorowski (1988) Nr. 297 .

Vgl. Körber (1998) 67; Petersohn (1963) 140. 
mae eine Gemeinschaft zwischen den Ratsherrn der Städte Königsberg und den Angehörigen der Universität. ${ }^{36}$ Er unterbreitet den intendierten Rezipienten ein Identifikationsangebot, um auf diese Weise ihre Gunst zu gewinnen und die Finanzierung der Albertina zu sichern.

\section{Inhaltliche, formale und mediale Aspekte des Carmen: Eine hybride Gattung}

In dem Ausgangstext, der Thomaes Übertragung zugrunde liegt, wird das Thema des Neids umfassend beleuchtet. ${ }^{37} \mathrm{Zu}$ Beginn seiner Predigt beschäftigt sich Basilius mit der Ursache dieser Leidenschaft. Er unterscheidet zwischen Gut und Böse, Gott und Teufel, um den Neid direkt dem Satan zuzuordnen. Anschließend stellt er die Auswirkungen dieses Übels, das er für das größtmögliche hält, dar. Für den Betroffenen gebe es keine schlimmere Krankheit, da der Neid die eigene Seele schmerzlich verwunde, ohne dass dieses Leiden offen zugegeben und ärztlich behandelt werden könne. Nur im Unglück der anderen finde der Neidische Heilung. Die schrecklichen und verwerflichen Handlungsweisen, zu denen der Neid führt, veranschaulicht Basilius an mehreren Beispielen des Alten und Neuen Testaments. Er verweist auf die ungerechte Behandlung Davids durch Saul, den Verkauf Josephs durch seine eifersüchtigen Brüder und die Auslieferung des Erlösers durch die christusfeindlichen Juden. Als angemessene Reaktion wird empfohlen, sich von Neidern fernzuhalten, auch wenn sich diese mit ihrem Verhalten, wie der Kirchenvater betont, selbst am meisten schadeten. Zugleich bietet er den Betroffenen Hilfe an und zeigt, wie sie ihre Leidenschaft durch Gleichgültigkeit in materiellen Angelegenheiten überwinden können. Dass Lebensgüter keinen Anlass bieten sollten, Neid zu empfinden, versucht Basilius argumentativ zu belegen, indem er zwei Alternativen aufzeigt: Weder sei jemand zu beneiden, der seinen Reichtum eigennützig einsetze, noch derjenige, der ihn sinnvoll zu gebrauchen wisse. Im ersten Fall führe der Reichtum nämlich ins Verderben, während er im zweiten Fall allen zu Gute komme. Den Besitzenden rät Basilius zu einer tugendhaften Gesinnung, die vor Neid schütze, statt ihn durch dummen Stolz zu schüren.

$3^{6} \quad \mathrm{Zu}$ der Strategie, sich von den lasterhaften Anderen abzugrenzen, um die eigene Leserschaft als wahrhaft christliche zu definieren, vgl. auch Toepfer (2010); Vessey (1997) $83^{2}$.

37 Eine Identifikation der benutzen Vorlage war nicht möglich. Die jüngste deutsche Übersetzung der Predigt stammt von Stegmann, vgl. dessen Ausgabe Basilius von Cäsarea (1925). 
Nachdrücklich warnt er auch vor dem Übel der Heuchelei und stellt die negativen Folgen vor Augen, die bei einem solchen Verhalten drohen: Das ewige Seelenheil kann auf diese Weise verspielt werden.

Josua Thomae folgt bei seiner Übertragung dem Argumentationsgang des Basilius, lehnt sich in vielen Passagen inhaltlich eng an seine Vorlage an und gibt sie sinngemäß wieder. ${ }^{38}$ Zudem ergänzt er jedoch vorgegebene Beispiele, illustriert bestimmte Bemerkungen und fügt kleine Explikationen und ganze Exkurse ein. Dabei handelt es sich nicht etwa um aktuelle Bezugnahmen, mit denen die Warnungen vor dem Neid im Sinne der lokalen bildungspolitischen Auseinandersetzung interpretiert werden könnten. Vielmehr steigert Thomae die Zahl der rhetorischen Stilmittel und der intertextuellen Anspielungen auf die antike Literatur, die sich in der Predigt des Kirchenvaters finden. So verweist der Königsberger Interpret auf das traurige Beispiel des Bettlers Iros auf Ithaka, ${ }^{39}$ der im Haus des Odysseus keinen zweiten Almosenempfänger dulden will und von dem heimgekehrten Hausherrn eine verdiente Strafe für seine Missgunst erhält. ${ }^{40}$

Ebenso bedient sich Thomae der Stoffgeschichte der homerischen Epen, um die gefährliche Wirkung von Heuchelei und Neid zu veranschaulichen. Er übernimmt zunächst den Vergleich des Basilius, dass im Inneren verborgene Kämpfe einem Menschen ebenso zum Verhängnis werden könnten, wie unter der Meeresoberfläche verborgene Felsen einen unvorsichtigen Schifffahrer ins Verderben stürzten. Anschließend fügt der Übersetzer einen eigenen Vergleich hinzu, der sich auf die List des Odysseus bei der Zerstörung Trojas bezieht. Er erklärt, dass sich durch das verwerfliche Laster des Neids ebenso viele Mengen an Übel ausbreiten würden, wie einst bewaffnete Männer dem hölzernen Pferd im Inneren der Stadt Troja entstiegen seien. ${ }^{41}$ Mit diesen antiken Allusionen

38 Zu der Übersetzungspraxis allgemein vgl. Bussmann, Hausmann u. a. (2005); Guthmüller (1995); Heinzle, Johnson, und Vollmann-Profe (1996).

39 Vgl. Thomae (1580) Sign. Dib: „Nanque iniustitiae si sunt ea uincula tantum: / Praeditus his misero quid non est tristius Iro?“ Übers.: „Denn wenn diese Fesseln der Ungerechtigkeit so stark sind, was ist weniger traurig, als durch diese gefesselt zu sein wie der arme Iros?"

$40 \quad$ Vgl. Von der Mühll (1993) 1-107.

41 Thomae (1580) Sign. D2b: „Aequoreis ceu tectus aquis lapis undique, nauem, / Quam Dijs, quam uotis linquens, sua frena remisit / Rector, in undisoni demergit tartara ponti. / Ex detestando quare cum tanta malorum / Agmina promanant Liuore, quot arduus astans / Armatos Troiae medijs in moenibus olim / Fudit equus, tenuis quot piscibus unda natatur." Übers.: „Gleichwie ein Meeresfelsen, der von allen Seiten von Wasser bedeckt wird, ein Schiff, dem der Steuermann, es den Göttern wie den Gebeten anheimstellend, seine Führung überlässt, in die Unterwelt des wellenrauschenden Meeres herabzieht. Deshalb ist der Neid zu verwünschen, weil aus ihm so viele Mengen an Übel hervorgehen, wie das 
verleiht Josua Thomae seinem Carmen nicht nur eine gesteigerte humanistische Dignität und erhöht das Anspruchsniveau des Ausgangstextes, sondern stellt auch seine eigene Gelehrsamkeit unter Beweis. Die Übertragung des Studenten Thomae widerlegt damit zweifelsfrei die in Königsberg und Umgebung kursierenden Verleumdungen, die Lernenden der Albertina verfügten über so geringe lateinische Sprachkenntnisse, dass die Schüler im Pädagogium nicht einmal mensa deklinieren könnten. ${ }^{42}$

So gut die Oratio Über den Neid inhaltlich geeignet ist, um sie in einer finanzpolitischen Kontroverse zu instrumentalisieren, so wenig stimmen die formalen Charakteristika des Ausgangstextes mit denen eines Carmen überein. Wie alle Predigten sind auch die Homilien des Basilius Magnus, der von den Humanisten wegen seiner Eloquenz und Gelehrsamkeit hochgeschätzt wird, ${ }^{43}$ in Prosa abgefasst, sei es um den religiösen Wahrheitsgehalt zu betonen oder um die natürliche Art menschlichen Redens beizubehalten. Dabei orientiert sich die Homiletik an den Regeln der klassischen Rhetorik, um die Gläubigen in gewünschter Weise beeinflussen zu können. ${ }^{44}$ Mit Hilfe sprachlicher Bilder, detaillierter Schilderungen sowie von Vergleichen und Exempeln soll das katechetische und moralische Wissen anschaulich vermittelt werden. Eine klare Disposition und eine schlüssige Argumentationsfolge dienen dazu, das Verständnis zu fördern, wobei Kernaussagen durch den Bezug auf die Heilige Schrift theologisch legitimiert werden. Sprachliche Figuren, wie rhetorische Fragen, Interjektionen und Appelle, helfen, die Eingängigkeit der Rede zu verstärken. Mit diesen Stilmitteln wird die mündliche Vortragsform imitiert und inszeniert, die auch bei der schriftgebundenen Lektüre ihre Wirkung entfaltet. Weil die Predigt zwischen Mündlichkeit, Bildlichkeit und Schriftlichkeit changiert, kommt ihr ein eigener medialer Status zu. ${ }^{45}$

Viele dieser medialen Charakteristika einer Homilie behält Josua Thomae bei, wenn er die Predigt des Basilius in Hexameter verwandelt. Indem sich nun ein Sprecher-Ich an ein Publikum wendet, wird die Darstellungsweise in einer Art personalisiert, die einem Carmen heroicum gewöhnlich fremd ist. Statt einem anonymen Publikum in formelhafter Sprache distanziert von einem Geschehen zu berichten, wird eine Gesprächssituation simuliert, in der die Rezipienten einbezogen sind:

aufrecht stehende, hochragende Pferd einst bewaffnete Männer innerhalb der Mauern der Stadt Troja aussteigen ließ, so viele wie ein seichtes Gewässer Fische in sich schwimmen lässt."

$42 \quad$ Vgl. Von Selle (1956) 71.

43 Vgl. Toepfer (2007) 194-205.

44 Zur Abhängigkeit der Homiletik von der rhetorischen Texttheorie vgl. auch Sieveke (1974).

45 Grundlegend zu dieser Thematik ist der Sammelband Wetzel und Flückiger (2010). 
Ergo quid? An satis est scelus hoc taxasse nefandum? / [...] Iamque aliquis dicat: modo qua ratione cauendum Ne cruciet saeuo pestis nos ista ueneno? ${ }^{46}$

Immer wieder werden die imaginierten Zuhörer mit eindringlichen Fragen zum Nachdenken aufgerufen - „Dic mihi terribilis quid dicet in aethere iudex / Cum freta, cum tellus, fulgensque peribit Olympus? ${ }^{447}$ - und zu einem gottesfürchtigen und frommen Handeln angehalten:

Nempe ut sis iustus, prudens, mentisque benigna

Temperie, fortis casus perferre malignos

Et patiens, sanctae decus ob pietatis amicum.

Te seruare etenim potes hac ratione, tibique

Insignem summa laudem uirtute mereri. ${ }^{48}$

Die zitierten Verse zeigen, dass Josua Thomae die Paränese des Kirchenvaters in seiner Adaptation in ein heroisch-episches Sprachgewand kleidet. ${ }^{49}$ Auf diese Weise ist ein Werk entstanden, das als hybrid bezeichnet werden muss, weil es Merkmale unterschiedlicher Textsorten beinhaltet: Sein paränetischer Inhalt und alle rhetorischen Figuren, die sich an ein imaginiertes Publikum richten, offenbaren die Herkunft des Carmen aus dem Bereich der Homiletik. Sein Versmaß und die hinzugefügten Anspielungen auf die antike Literatur und Mythologie kennzeichnen den Text dagegen als episches Gedicht.

Wie für die Predigt ist ebenso für das Epos eine spezifische Form von Medialität kennzeichnend, die in der Schriftlichkeit Elemente der Mündlichkeit auf-

46 Thomae (1580) Sign. C4a. Übers.: „Was nun? Genügt es etwa diesen gottlosen Frevel richtig eingeschätzt zu haben? [...] Schon könnte einer sagen: Auf welche Weise und mit was für einer Einstellung ist dies zu vermeiden, damit diese Pest uns nicht mit ihrem schlimmen Gift peinigt?"

47 Thomae (1580) Sign. Dia. Übers.: „Sag mir, was mag der Ehrfurcht gebietende Richter im Jenseits sagen, wenn die Meere, wenn die Erde und der Himmel vergangen sind?" Rhetorische Fragen durchziehen den gesamten Text, vgl. auch Sign. A4a, B2a, B3a, B4, C1, C2a, $\mathrm{C}_{3} \mathrm{~b}, \mathrm{C}_{4}$ und Dib.

48 Thomae (1580) Sign. D2a. Übers.: „Denn wenn du gerecht, klug und durch gütige Selbstbeherrschung des Geistes stark bist, Unglücksfälle zu ertragen, und geduldig wegen der freundlichen Ehre der heiligen Frömmigkeit, dann nämlich kannst du dich durch diese Gesinnung retten und für dich unermesslichen Ruhm durch die höchste Tugend verdienen."

49 Auerbach (2001) 147 stellt heraus, dass die in der antiken Rhetorik streng getrennten Stilarten, sermo gravis und sermo humilis, im Christlichen von vornherein verschmolzen sind, wobei er insbesondere auf die Inkarnation und die Passion Christi verweist. 
weist. Da der homerische Hexameter einer mündlichen Vortragssituation entstammt, gilt er mitsamt seiner festgefügten sprachlichen Wendungen als Signum der Mündlichkeit, unabhängig von der konkreten Entstehung, Überlieferung und Rezeption eines Epos..$^{50}$ Dem antiken Versmaß wohnt eine besondere Dynamik inne, die sowohl bei einem memorialen als auch bei einem schriftgestützten Vortrag oder gar bei der Lektüre zum Tragen kommt. Daher wird der eigentümliche Status zwischen Mündlichkeit und Schriftlichkeit, der für die antike Oratio des Basilius charakteristisch ist, in dem frühneuzeitlichen Carmen nicht nur beibehalten, sondern durch die metrische Übertragung sogar potenziert. Indem Thomae von zwei verschiedenen Gattungen Anleihen nimmt, die beide aus einer Vortragssituation erwachsen sind und diese im Medium der Schrift konzeptionell verankern, wird die Mündlichkeit in seinem Text in zweifacher Weise inszeniert. An einigen Stellen wird der Eindruck erzeugt, als ob ein antiker Rhapsode vor einem christlichen Publikum einen Beichtspiegel und Tugendkatalog vorträgt.

Mit der metrischen Überformung der Predigt sind jedoch auch Veränderungen hinsichtlich der dem Text eingeschriebenen Performativität verbunden, wie der Schluss des Drucks zeigt. ${ }^{51}$ Thomae übernimmt in seiner Übersetzung zwar den Lobpreis Gottes, mit dem Basilius seine Rede beendet hat, geht aber nicht mehr von der andächtigen Anteilnahme der Rezipienten aus. Anstelle der Schlussformel der Homilie, mit der die Gläubigen zum performativen Akt angeleitet werden, ${ }^{52}$ ist in dem Druck nur das Ende des Carmen markiert: Vt peccata Deus fert nostra ingentia mitis. / Cui laus, cui uirtus, cui gloria digna triumpho /Et simul aeternos fama est mansura per annos. / FINIS. ${ }^{53}$ Das gemeinsame ,Amen' des Gebets weicht dem ,Finis' der Argumentation. Selbst wenn

50 Vgl. Latacz (2003) 11-13; Seeck (2004) 31. In der Oral poetry-Forschung wurden diese Charakteristika als Indiz für die Mündlichkeit der Epen gewertet (vgl. v.a. Parry (1930)). In der klassischen Philologie wird diese Theorie heute sehr kritisch gesehen (vgl. Seeck (2004) 51-52; Latacz (2003) 13, 26-30; Goody (1977)), weil die daraus abgeleitete Auffassung von der archaischen Fremdheit Homers eine Würdigung der poetischen Qualitäten seiner Epen verhindert hat. Aufgrund des hohen inneren Organisationsgrads der Dichtung und der starken Standardisierung des Formelschatzes gilt die Gleichsetzung von Formelhaftigkeit und Mündlichkeit als verfehlt.

51 Zum Begriff der Performativität vgl. Velten (2002). Zur Bedeutung des Performativen für die Medialitätsforschung vgl. Arbeitsgruppe Medien (2004); Eming, Lehmann, und Maassen (2002); Krämer (2002; 2004).

Zur Aktivierung der Rezipienten durch Gebetsformeln vgl. Herberichs (2007) 178-180; Toepfer (2009) 124-13o.

53 Thomae (1580) Sign. D3a. Übers.: „damit Gott Gnade gegenüber unseren unermesslichen Sünden walten lässt. Ihm sei Lob, ihm sei Ruhm, ihm sei würdige Ehre in Herrlichkeit, und sein Preis wird ebenso fortwähren bis in Ewigkeit. Ende.“ 
sich dieses Signalwort primär an Leser richtet, ist daraus nicht abzuleiten, dass Thomaes Version ausschließlich für die Lektüre bestimmt ist. Der beobachtete Verzicht auf die Gebetsformel erlaubt keineswegs, Vokalität und Oralität als verschiedene Rezeptionsformen von Oratio und Carmen gegeneinander zu stellen. ${ }^{54}$ Vielmehr sollte der Schluss als Indiz für die unterschiedlichen Rezeptionskontexte von Ausgangs- und Zieltext gewertet werden, bei denen sich der Akzent von einem genuin religiösen zu einem politisch-sozialen Verwendungszweck verschiebt. Auf welche Weise Thomaes Übertragung rezipiert und verbreitet wurde, so dass mit ihr in der Königsberger Bildungskontroverse publikumswirksam Stellung bezogen werden konnte, lässt sich mit Hilfe der Überlieferungsgeschichte erhellen.

Das Carmen heroicum, das Josua Thomae auf der Grundlage der Predigt De invidia des Kirchenvaters Basilius gestaltet hat, ist unikal überliefert. Der Druck, der 1580 bei dem Königsberger Universitätsdrucker Georg Osterberger erschien, befindet sich heute im Besitz der Herzog August Bibliothek Wolfenbüttel und ist in einem zeitgenössischen Sammelband mit 56 weiteren Drucken zusammengebunden. ${ }^{55}$

Schon die auffallend hohe Anzahl an Beibänden, die zum überwiegenden Teil in der Stadt am Pregel veröffentlicht wurden, lässt auf den geringen Umfang der einzelnen Exemplare schließen. Sämtliche Drucke würdigen in der Form von Hochzeits-, Glückwunsch-, Promotions-, Abschieds- und Leichengedichten lokale Persönlichkeiten und lassen sich somit dem Bereich der Gelegenheitsdichtung zuordnen. ${ }^{56}$ Den Band eröffnet ein Klagelied, das anlässlich der Beerdigung von Catharina, der Ehefrau Peter Sicks, verfasst und 1575 gedruckt wurde..$^{57}$ Den Abschluss bilden drei Drucke aus dem Jahr 1584: ein Carmen gratulatorium, mit dem Joachim Cimdarsus den Markgrafen Georg Friedrich von Brandenburg beglückwünscht, ein Xeniolum (,Gastgeschenk'),

54 Den Begriff der Vokalität führte Ursula Schaefer, anlehnend an Paul Zumthor (1987) 21, ein, um auf diese Weise die dem Text eingeschriebene Stimme des Erzählers in der altenglischen Heldendichtung zu bezeichnen. Vgl. Schaefer (1992), bes. 114-118.

55 Vgl. Anhang, Nr. 19.

$5^{6}$ Systematisch erfasst werden solche Drucke im Handbuch des personalen Gelegenheitsschriftums in europäischen Bibliotheken und Archiven, im Zusammenwirken mit der Forschungsstelle Literatur der Frühen Neuzeit und dem Institut für Kulturgeschichte der Frühen Neuzeit der Universität Osnabrück (Garber (2001ff.)).

Vgl. Anhang, Nr. 1. 
das Georg Poltzin Andreas Iridus widmet, und verschiedene Carmina nuptialia, in denen der Hochzeit von Burchard Harbarth und Concordia, der Tochter des Andreas Funcius, gedacht wird. ${ }^{58}$ Von dem Übersetzer des Basilius, Josua Thomae, stammen in dem Sammelband insgesamt vier Beiträge: Neben dem Carmen heroicum, Über den Neid' finden sich ein Ode zum Osterfest und zwei Hochzeitsgedichte, von denen Thomae das eine anlässlich der Eheschließung von Caspar Otto Etzel mit Ursula, der Tochter Johann Korns, und das andere anlässlich der Vermählung des Magisters Martin Winter mit Barbara Cramer verfasst hat. ${ }^{59}$

Mehrere Exemplare des Sammelbandes, zu denen auch die Übertragung des griechischen Kirchenvaters zählt, sind ausdrücklich für einen bestimmten Empfänger gedacht. Das Titelblatt von De invidia ist mit einem handschriftlichen Vermerk versehen, durch den der Druck Ornatissimo ac prudentissmo uiro Magistro Laurentio Panteno, graecae linguae in Academia Regiomontana professori, zugeeignet wird (vgl. Abb. 1). Obwohl der Name des Spenders nicht genannt wird, ist davon auszugehen, dass es sich um eine Widmung Thomaes handelt, der seinem Griechisch-Lehrer an der Albertina ein Exemplar seiner Version überreicht hat. Noch sieben weitere Drucke des Sammelbandes sind Laurentius Pantenus handschriftlich zugeeignet, ${ }^{60}$ des Weiteren findet sich an anderer Stelle ein eigener Besitzeintrag ${ }^{61}$ und schließlich wird in einem Druck die Hochzeit des Königsberger Professors mit Esther Dabbertana gefeiert. ${ }^{62}$ Diese Beobachtungen legen den Schluss nahe, dass der in der Herzog August Bibliothek aufbewahrte Band 49.1 Poetica ursprünglich Pantenus gehörte. Demnach hat der Professor der Albertina eine persönliche Sammlung von Kasualgedichten angelegt, die in einer weitgehend chronologisch geordneten Reihenfolge die sozialen Ereignisse der Königsberger Oberschicht in den Jahren 15751584 widerspiegeln. ${ }^{63}$

Für die Rekonstruktion der Rezeption und die Frage nach dem Grund für die metrische Übertragung ist dieser überlieferungsgeschichtliche Befund von entscheidender Relevanz. Wenn Pantenus die Predigt De invidia in seine Sammlung an Gelegenheitsdrucken aufgenommen hat, bedeutet dies, dass für ihn

\footnotetext{
$5^{8}$ Vgl. Anhang, Nr. 55-57.

59 Vgl. Anhang, Nr. 19, 32, 33 und 44.

6o Vgl. Anhang, Nr. 1, 13, 16, 22, 26, 42 und 53 .

61 Vgl. Anhang, Nr. 31.

62 Vgl. Anhang, Nr. 34.

63 Zu der Verortung der Gelegenheitsdichter im Kontext der Universität und der Adressaten im städtischen Bürgertum, vgl. Walter (2005) 51-52. Die meisten seiner Beobachtungen, die sich vor allem auf die Kasualia des 17. Jahrhunderts stützen, gelten bereits für die Drucke des hier vorgestellten Sammelbandes.
} 
kein Unterschied zwischen den einzelnen Königsberger Bürgern gewidmeten Gedichten und der den Ratsherren der drei Städte zugeeigneten Übersetzung des Kirchenvaters besteht. ${ }^{64}$ Ermöglicht wird diese Zuordnung des Textes vor allem durch seine formale Gestaltung als Carmen heroicum, wohingegen der paränetische Inhalt und die Stilelemente der Homilie für den zeitgenössischen Rezipienten nicht ins Gewicht zu fallen scheinen. Zudem weist Thomaes Übersetzung des Basilius ein weiteres wesentliches Merkmal auf, das für Kasualdichtung charakteristisch ist: Die Carmina werden stets zu einem konkreten Anlass verfasst. ${ }^{65}$ Auch hinsichtlich seiner aktuellen Bezugnahme ist Thomaes Version anderen Gedichten vergleichbar, die Ereignisse von gesellschaftlichem Rang literarisch in Szene setzen.

Der überlieferungsgeschichtliche Befund erlaubt, die Forschungserkenntnisse über die Funktion und Bedeutung von Gelegenheitsgedichten auch auf De invidia zu übertragen. ${ }^{66}$ Es ist davon auszugehen, dass Thomaes Übertragung in ähnlicher Weise rezipiert wurde wie andere Kasualgedichte, die der Königsberger Elite mündlich vorgetragen und durch den Druck auch außerhalb der Stadt verbreitet wurden. ${ }^{67}$ Die Carmina sollten die öffentliche Bedeutung der Geehrten bestätigen und vermehren, ihren Ruhm sichern und der Nachwelt bewahren. Die Gedichte sorgten für die Unterhaltung der Gäste bei einem

64 Da sich die Forschungsprojekte zu den Königsberger Drucken verschiedenen Textsorten widmen, sei es der Gelegenheitsdichtung oder genuin akademischem Schrifttum wie Vorlesungsverzeichnissen, Disputationen oder Dissertationen, wird die Möglichkeit einer systematischen Differenzierung suggeriert, die sich in der Praxis nicht immer aufrechterhalten lässt. Zu den akademischen Schriften vgl. v. a. Komorowski und Marti (2004).

65 Grundlegend für den Begriff und die Gattung der Gelegenheitsdichtung ist die Habilitationsschrift Segebrecht (1977). Er kritisiert die bisherige Abwertung der Kasualcarmina in der Forschung als „Machwerk“, wohingegen die zweckfreien Erlebnisgedichte als „Kunstwerk" gewertet würden (vgl. S. 1-5). Segebrecht identifiziert vier konstituierende Faktoren für die Kasuallyrik, die sich alle auch in Thomaes Carmen heroicum feststellen lassen: Gelegenheit, Gedicht, Autor und Adressat (vgl. S. 68-73). Legitimiert wird die Anwendung des Begriffs „Kasualgedicht“ für Thomaes Carmen auch durch die Verfahrensweise der Herausgeber des Handbuchs des personalen Gelegenheitsschrifttums, in das Widmungsgedichte aus Dissertationen oder anderen Werken aufgenommen werden (vgl. Walter 2004; 2005, 50) und in dem sich auch ein Carmen heroicum findet (Bd. 16, Nr. 714). Einen kurzen Überblick über die Gattung gibt auch der von Segebrecht verfasste Artikel im Reallexikon der Literaturwissenschaft. Vgl. Segebrecht (1997).

66 Segebrechts Untersuchung hat der geschmähten Gattung des Gelegenheitsgedichts wieder zum Ansehen verholfen und den Anstoß zu weiteren Studien gegeben. Vgl. z. B. Adam (1988). Durch das Handbuch des personalen Gelegenheitsschrifttums wird es immer besser möglich, das umfangreiche Quellenmaterial zu überschauen und auszuwerten.

67 Zur Publikation von Gelegenheitsgedichten vgl. Segebrecht (1977) 191-193; Segebrecht (1976). 
bestimmten Anlass, hielten später die Erinnerung an das gefeierte Ereignis wach und vermittelten bei Abwesenheit Informationen darüber. ${ }^{68}$ Sämtliche sozialen Funktionen, die die Kasualdichtung in diesem Kontext übernimmt, werden auch von Thomaes Übersetzung erfüllt:69 Das Carmen heroicum dient der Repräsentation, indem der Einsatz der Ratsherrn für die Bildung gerühmt und der gelehrte Status der Universitätsangehörigen herausgestellt wird. Eine Selbstvergewisserung und Festschreibung von sozialen Normen erfolgt, wenn Thomae die politische Führungsschicht an ihr bisheriges bildungsfreundliches Verhalten erinnert, vor den drohenden Gefahren bei einer Änderung warnt, gegen Verleumdungen Stellung bezieht und eine Fortsetzung der finanziellen Förderung zu erreichen sucht. Der Druck des Carmen verhindert sein Vergessen und überführt den konkreten Anlass, die problematische Lage der Albertina, ins kollektive Gedächtnis der Stadt, wobei die lyrische Form die gemeinschaftliche Rezeptionssituation präsent hält und die Performativität dem Text einschreibt.

Die Sammlung des Laurentius Pantenus zeigt, dass das Carmen heroicum in dem Sinne verstanden und rezipiert wurde, wie Josua Thomae dies in seinem Widmungsschreiben vorgab: als ein aktueller Beitrag zu einem Ereignis von gesellschaftlicher Bedeutung, dem Finanzierungsnotstand der Universität. Der überlieferungsgeschichtliche Befund stützt und vertieft nicht nur die bisherige Interpretation, sondern bietet auch eine Antwort auf die Frage nach dem „Warum“ einer Versübersetzung, die für die Wirkungsgeschichte des griechischen Kirchenvaters im Humanismus und der Reformationszeit so ungewöhnlich ist: Mit Hilfe der Metrik wird die thematisch passende Predigt, durch die die Kritik an der Universität als Verleumdung zurückgewiesen werden kann, in ein Kasualgedicht verwandelt. Diese Transformation ermöglicht es Josua Thomae, an lokale Dichtungstraditionen anzuknüpfen und erprobte Vermittlungsformen in Anspruch zu nehmen. ${ }^{70}$ Als Carmen heroicum werden die Warnungen des Kirchenvaters vor dem äußerst verwerflichen Laster des

68 Zu den Produktions- und Rezeptionsmotivationen von Kasualcarmina vgl. v. a. Segebrecht (1977) $174-188$.

69 Vgl. Drees (1986), bes. S. 456-461; Freise (2004), bes. 249-250, 268.

70 Zu der Dominanz des Kleinschrifttums und der lokalen Ausrichtung der Königsberger Drucker vgl. Walter (2005) 49. Aufgrund der schlechten Überlieferungsbedingungen sind das akademische Kleinschrifttum und die Gelegenheitsdichtung bislang nur sehr unvollständig erfasst und erschlossen. Die Tradition der Gelegenheitsgedichte setzte in den Anfangsjahrzehnten des Königsberger Buchdrucks ein; sie war von Anfang an mit der Universität und dem herzoglichen Haus verbunden und erstreckte sich noch im 16. Jahrhundert auf das städtische Bürgertum. Ihre große Zeit erlebte die Königsberger Gelegenheitsdichtung im 17. Jahrhundert mit Simon Dach. 
Neids, dessen sich die Bildungsgegner des ersten und zweiten Stands schuldig gemacht haben, in einen städtischen Rezeptionskontext überführt und können sowohl im mündlichen Vortrag als auch in gedruckter Form verbreitet werden. Somit ist die Metrik das entscheidende Medium, um die Predigt des Basilius Magnus in der Königsberger Bildungskontroverse um 1580 öffentlichkeitswirksam zu instrumentalisieren.

\section{Anhang}

Auflistung der Drucke des Sammelbandes

Wolfenbüttel, Herzog August Bibliothek, A: 49.1 Poetica

1. Fabricius, Lorenz. 1575. Threnus hymenis conubialis in funere Catharina coniugis Petri Sickij. Danzig: Jakob Rhode. (vD 16 F 441)

2. Frum, Johannes. 1576. Carmen scholasticos gradus a virulentis anabaptistarum morsibus vindicans. Königsberg: Georg Osterberger. (VD 16 F 3178)

3. Smidenstedt, Hartwig. Um 1575. In gloriosam resurrectionem Iesu Christi. [o. O.].

4. Wapner, Lazarus. 1576. Scriptum ad Melchiorem Thierbachium et Ioannem Wapnerum. Königsberg: Georg Osterberger.

5. Francke, Heinrich. 1576. Carmen gratulatorium in honorem Georgii Schepeleri, cum decerneretur ei gradus Magisterij. Königsberg: Georg Osterberger. (VD 16 F 2177)

6. Weis, Paulus. 1577. Carmen in festo pentecostes. Königsberg.

7. Weis, Paulus. 1578. Carmen in festo paschatis. [o.O.].

8. Schreck, Valentin. 1579. Epithalamion in nuptiis Constantini iunioris, Constantini Ferberifilij, et Elisabethae, Hermanni Hackii filiae. [Danzig: Jakob Rhode]. (VD 16 S 4093)

9. Schreck, Valentin. 1579. Elegidion ad Gerhardum Iohannis Brandes senioris filium pro felici nuptiarum auspicio cum Catharina Ioannis Cremeri filia. Danzig: Jakob Rhode. (VD 16 S 4095)

10. Schrön, Johannes. 1579. Ode Saphica in gratiam Ulrici Gutzmeri, gradu Magisterij ornati et promoti. [o.O.].

11. Hesshusen, Gottfried, und Hesshusen, Heinrich. 1579. Carmina gratulatoria in honorem Iohannis Olearii, cum foedere iugali Anna Tilemani Heshusii filia iungeretur. Helmstedt: Jakob Lucius. (vD 16 H 2973)

12. Weis, Paul. 1579. Carmen in festo pentecostes. [0.O.].

13. N.N. 1579. Carmen gratulatorium in adventum Martini Berzovicz. Königsberg: Georg Osterberger.

14. Titius, Simon. 1573. Oratio De Officio Et Lavdibvs Principis Boni, Habita Cvm 
Qvintvm Perentaretvr Illvstrissimo Optimoquae beatae memoriae Principi Alberto Marchioni Branderburgensi etc. Duci Prussiae primo. Königsberg: Hans Daubmann.

15. Winter, Martin. 1579. Theses ethicae. [o. O.].

16. Hartmann, Sebastian. 1579. Epithalamion in honorem Matthiae Stoii et Elisabetae Sebastiani Langen filiae. Königsberg: Georg Osterberger.

17. N.N. 1580. Epithalamia in honorem nuptiarum Friderici à Nostitz et Annae Christophori à Saleth filiae. Königsberg: [Georg Osterberger]. (VD 16 E 1778)

18. Poltzin, Georg. 1580. Threnodia Iesu Christi. Königsberg: [Georg Osterberger]. (vD 16 P 4074)

19. Thomae, Josua. 1580. Oratio D. Basilii de nocentissimo invidiae vicio, carmine heroico translata et illustrata. Königsberg: Georg Osterberger. (VD 16 B 716)

20. (Weis, Paul) 1580. Epicedia in obitum Michaelis Nesseni. Königsberg: Georg Osterberger. (VD 16 W 1561)

21. Brettschneider, Johannes. 1580. Carmen in honorem Henrici Sprengelii et Catarinae Martini Fuchs filiae nuptias. Königsberg: Georg Osterberger. (VD 16 B 8019)

22. Cimdarsus, Joachim. 1580. Carmen panegyricum in felicem natalem Alberti II. Königsberg: Georg Osterberger. (VD 16 C 3913)

23. Cimdarsus, Joachim. Um 1580. Nuptiis Michaelis Gisii et Ursulae Heidensteiniae. [o.O.]. (VD 16 C 3920 )

24. Weis, Paul. 1580. In festo paschatis. [o. O.].

25. (Weis, Paul). 1580. Epicedia in obitum Michaelis Nesseni. Königsberg: Georg Osterberger. (VD 16 W 1561)

26. N.N. 1580. In honorem et memoriam Christophori Heilsbergeri ex academia Francofordiana alio discedentis carmina. Frankfurt a. O.: Andreas Eichorn.

27. Weis, Paul. 1580. Carmen in natalem Christi scriptum. [o. O.].

28. Weis, Paul. 1580. In funere Michaelis Sickermanni. Königsberg: Georg Osterberger. (VD 16 W 1562)

29. Wolf, Samuel. 1581. Aenigma denotans annum nuptiarum Martini Winteri cum Barbara Christophori Cramerifilia. [o. O.].

3o. Cimdarsus, Joachim. 1581. Elegia de puero Bifronte. [o. O.].

31. Schreck, Valentin. 1581. Epithalamion in nuptiarum celebratione Ioannis Borchmanni et Catharinae Schutziae. Danzig: Jakob Rhode. (VD 16 S 4094)

32. Thomae, Josua. 1581. Ode sapphica in nuptias Casparis Ottonis Etzel et Ursulae Iohannis Korn. Königsberg: Georg Osterberger.

33. (Thomae, Josua). 1581. Carmina gratulatoria nuptiis M. Martini Winteri et Barbarae Cramerianae. Königsberg: Georg Osterberger. (VD 16 T 992)

34. Poltzin, Georg. 1581. Nuptiis Laurentii Pantheni et Estherae Dabbertanae. Königsberg: [Georg Osterberger]. (VD 16 P 4072)

35. Weis, Paul. 1581. Carmen de nativitate Iesu Christi. [o. O.]. 
36. N.N. 1581. Nuptiis Danielis Poltzinii et Margarethae Petermans epithalamia. Königsberg: Georg Osterberger. (VD 16 N 2114)

37. Schreck, Valentin. 1581. Elegia nuptialis Martino Wintero celebranti ritus nuptiarum cum Barbara Christophori Crameri filia. Danzig: Jakob Rhode. (VD 16 S 4085)

38. Poltzin, Georg. 1582. Egloga picturae Christophori allegoriam exponens. Königsberg: Georg Osterberger. (VD 16 P 4071)

39. N.N. 1582. Ad Stephanum I. post partam victoriam redeuntem. Königsberg.

40. Cimdarsus, Joachim. 1582. Epicedion Mariae, principis Guilielmi coniugi. Königsberg: Georg Osterberger. (VD 16 C 3919)

41. (Cimdarsus, Joachim). 1582. In honorem octo virorum. Königsberg: Georg Osterberger. (VD 16 C 3921)

42. Schreck, Valentin. 1582. Charites sive gratiarum actio pro liberali instauratione aedificii scholae Marianae Dantisci. Danzig: Jakob Rhode. (VD 16 S 4079)

43. Weis, Paul. 1581. Carmen in festo paschatis. [o. O.].

44. Thomae, Josua. Um 1580. Ode Sapphica de gloriosa Christi resurrection. [0. O.].

45. Poltzin, Georg. 1582. Nuptiis Simonis Crovii et Kopkianae Caspari Köpken filiae ac Davidis Poltzinii sponsi et Sophiae Lucianae filiae sponsae. Königsberg: [Georg Osterberger]. (VD $16 \mathrm{P} 4073$ )

46. Poltzin, Georg. 1582. Egloga picturae Christophori allegoriam exponens. Königsberg: Georg Osterberger. (VD 16 P 4071)

47. Wrassius, Daniel. 1579. Elegia gratulatoria ad Michaelem Lubenovium et Michaelem Nessenum. [o. O.].

48. Wolf, Samuel. 1581. Aenigma denotans annum nuptiarum Martini Winteri cum Barbara Christophori Crameri filia. [o. O.].

49. Cimdarsus, Joachim. 1583. Carmen funebre in memoriam Matthiae Stoii. [o. O.].

50. Schade, Paul. 1583. Ode Alcaica ad nuptias Vito Erasmo Tettelbachio. Nürnberg.

51. N.N. Nuptiis Casparis Perbandi et Catharinae Mauricii Wegneri filiae - unvollst.

52. Von Hogenkamp, Arnold. 1583 . Belgiadum sive heroidum epistolarum. Königsberg: [Georg Osterberger]. (VD $16 \mathrm{H}$ 4312)

53. Poltzin, Georg. 1584. Ecloga armaturam militis Christiani exponens. Königsberg: [Georg Osterberger]. (VD 16 P 4070)

54. Cimdarsus, Joachim. 1583. Elegidion in Georgii Friderici Marchionis Brandenburgensis. Königsberg.

55. Cimdarsus, Joachim. 1584. Carmen gratulatorium in Georgii Friderici Marchionis Brandenburgensis. Königsberg: Georg Osterberger.

56. [Poltzin, Georg]. 1584. Xeniolum Andreae Iridi. Königsberg: Georg Osterberger.

57. N.N. 1584. Carmina nuptialia in honorem Burchardi Harbarthi et Concordiae Andreae Funcii filiae. Leipzig: Georg Deffner. 


\section{Literatur}

Adam, W. 1988. Poetische und Kritische Wälder. Untersuchungen zu Geschichte und Formen des Schreibens , bei Gelegenheit'. Heidelberg (Beihefte zum Euphorion 22).

Arbeitsgruppe Medien. 2004. Über das Zusammenspiel von ,Medialität' und ,Performativität'. Paragrana. Internationale Zeitschrift für Historische Anthropologie 13:129185.

Asche, M. 2001. Frequenzeinbrüche und Reformen - Die deutschen Universitäten in den $1520 e r$ bis 156 oer Jahren zwischen Reformation und humanistischem Neuanfang. In Die Musen im Reformationszeitalter, hgg. von W. Ludwig. Leipzig (Schriften der Stiftung Luthergedenkstätten in Sachsen-Anhalt 1): 53-96.

Auerbach, E. 2001. Mimesis. Dargestellte Wirklichkeit in der abendländischen Literatur. Zehnte Ausgabe. Tübingen/Basel.

Backus, I. 1990. Lectures Humanistes de Basile de Césarée. Traductions Latines (14391618). Paris (Collection des Études Augustiniennes, Série Antiquité 125).

Basilius von Cäsarea. 1925. Über den Neid. In Basilius von Cäsarea. Ausgewählte Homilien und Predigten, hgg. von A. Stegmann, 289-30o. München (Bibliothek der Kirchenväter 47).

Besch, W. 1972. Vers oder Prosa? Zur Kritik am Reimvers im Spätmittelalter. In Festschrift für Hans Eggers zum 65. Geburtstag. Tübingen (= Рв в 94 [1972] Sonderheft): 745-766.

Brandstetter, A. 1971. Prosaauflösung. Studien zur Rezeption der höfischen Epik im frühneuhochdeutschen Prosaroman. Frankfurt am Main.

Bussmann, B., Hausmann, A. u. a. (Hg.) 2005. Übertragungen. Formen und Konzepte von Reproduktion in Mittelalter und Früher Neuzeit. Berlin/New York (Trends in Medieval Philology 5).

Daugsch, W. 1994. Die Universität Königsberg 1544-1945. In Die Albertina. Universität in Königsberg 1544-1994, hgg. von der Stiftung Gerhart-Hauptmann-Haus. Düsseldorf/Berlin: 9-54.

Dembowski, H. 2001. Zur Geschichte der Theologischen Fakultät der Universität Königsberg (1544-1945). In 450 Jahre Universität Königsberg. Beiträge zur Wissenschaftsgeschichte des Preußenlandes, hgg. von B. Jähnig. Marburg (Tagungsberichte der historischen Kommission für Ost- und Westpreußische Landesforschung 14): $25-38$.

Drees, J. 1986. Die soziale Funktion der Gelegenheitsdichtung. Studien zur deutschsprachigen Gelegenheitsdichtung in Stockholm zwischen 1613-1719. Stockholm.

Eming, J., A.J. Lehmann, und I. Maassen (Hg.) 2002. Mediale Performanzen. Historische Konzepte und Perspektiven. Freiburg im Breisgau (Rombach Wissenschaften: Reihe Litterae 97).

Erler, G. (Hg.) 1910-1917. Die Matrikel der Albertus-Universität zu Königsberg i. Pr. 3 Bd. Leipzig. 
Fink, G. (Hg.) 2003. Horaz, Epistulae. Lateinisch-deutsch, übers. von Gerd Herrmann. Düsseldorf (Tusculum Studienausgaben).

Freise, F. 2004. Das Kasualgedicht als öffentlicher Raum. Strategien der Repräsentation und sozialen Selbstvergewisserung in Thorner Gelegenheitsschriften des frühen 18. Jahrhunderts. In Offen und Verborgen. Vorstellungen und Praktiken des Öffentlichen und Privaten in Mittelalter und Früher Neuzeit, hgg. von C. Emmelius, F. Freise u.a. Göttingen: $249-268$.

Garber, K. (Hg.) $2001 \mathrm{ff}$. Handbuch des personalen Gelegenheitsschrifttums in europäischen Bibliotheken und Archiven, im Zusammenwirken mit der Forschungsstelle Literatur der Frühen Neuzeit und dem Institut für Kulturgeschichte der Frühen Neuzeit der Universität Osnabrück. Hildesheim/Zürich/New York.

Genette, G. 2001. Paratexte. Frankfurt am Main.

Goody, J. 1977. The Domestication of the Savage Mind. Cambridge.

Guthmüller, B. (Hg.) 1995. Latein und Nationalsprachen in der Renaissance. Vorträge des 37. Wolfenbütteler Symposions in der Herzog-August-Bibliothek Wolfenbüttelvom September 1995. Wiesbaden (Wolfenbütteler Abhandlungen zur Renaissanceforschung 17).

Heinzle, J., L.P. Johnson, und G. Vollmann-Profe (Hg.) 1996. Übersetzen im Mittelalter. Cambridger Kolloquium 1994. Berlin (Wolfram-Studien 14).

Henne, H. 1978. Literarische Prosa im 14. Jahrhundert - Stilübung und Kunst-Stück. ZfdPh 97: 321-336.

Herberichs, C. 2007. Lektüren des Performativen. Zur Medialität geistlicher Spiele des Mittelalters. In Transformationen des Religiösen. Performativität und Textualität im geistlichen Spiel, hgg. von I. Kasten und E. Fischer-Lichte. Berlin u.a. (Trends in Medieval Philology 11): 169-185.

Jähnig, B. (Hg.) 2001. 450 Jahre Universität Königsberg. Beiträge zur Wissenschaftsgeschichte des Preußenlandes. Marburg (Tagungsberichte der historischen Kommission für Ost- und Westpreußische Landesforschung 14).

Kellermann, K. (Hg.). 2004. Medialität im Mittelalter. Berlin (Das Mittelalter 9).

Kiening, C. 2007. Medialität in mediävistischer Perspektive. Poetica 39: 285-352.

Kleinschmidt, E. 1997. Autor. In Reallexikon der deutschen Literaturwissenschaft 1: 17618 o.

Komorowski, M. 1988. Promotionen an der Universität Königsberg 1548-1799. Bibliographie der pro-gradu-Dissertationen in den oberen Fakultäten und Verzeichnis der Magisterpromotionen in der philosophischen Fakultät. München u.a.

Komorowski, M. 2008. Die Universität Königsberg in der Frühen Neuzeit: Forschungsstand und perspektiven im Überblick. In Die Universität Königsberg in der Frühen Neuzeit, hgg. von H. Marti und M. Komorowski. Köln/Weimar/Wien: 1-26.

Komorowski, M., und H. Marti. 2004. Erfassung und Erschließung von Königsberger Universitätsschriften der Frühen Neuzeit - Eine Projektskizze. In Königsber- 
ger Buch- und Bibliotheksgeschichte, hgg. von A.E. Walter. Köln/Weimar/Wien (Aus Archiven, Bibliotheken und Museen Mittel- und Osteuropas 1): 787-8oo.

Körber, E.-B. 1998. Öffentlichkeiten der Frühen Neuzeit. Teilnehmer, Formen, Institutionen und Entscheidungen öffentlicher Kommunikation im Herzogtum Preußen von 1525 bis 1618. Berlin/New York (Beiträge zur Kommunikationsgeschichte 7).

Krämer, S. 2002. Sprache - Stimme - Schrift. Sieben Gedanken über Performativität als Medialität. In Performanz. Zwischen Sprachphilosophie und Kulturwissenschaften, hgg. von U. Wirth. Frankfurt am Main: 323-346.

Krämer, S. (Hg.) 2004. Performativität und Medialität. München.

Latacz, J. 2003. Homer. Der erste Dichter des Abendlands. Vierte Ausgabe. Düsseldorf/ Zürich.

Lawrynowicz, K. 1999. Albertina. Zur Geschichte der Albertus-Universität zu Königsberg in Preußen, hgg. von D. Rauschning. Berlin (Abhandlungen des Göttinger Arbeitskreises 13).

Leiner, W. 1965. Der Widmungsbrief in der französischen Literatur (1580-1715). Heidelberg.

Moeller, B. 2001. Die Universität Königsberg als Gründung der Reformation. In 450Jahre Universität Königsberg. Beiträge zur Wissenschaftsgeschichte des Preußenlandes, hgg. von B. Jähnig. Marburg (Tagungsberichte der historischen Kommission für Ost- und Westpreußische Landesforschung 14): 11-23.

Von der Mühll, P. (Hg.) 1993. Homer, Odyssea. Editio stereotypa editionis tertiae. Stuttgart, Leipzig (Bibliotheca scriptorum Graecorum et Romanorum Teubneriana 18)

Müller, J.-D. 1995. Auctor - Actor - Author. Einige Anmerkungen zum Verständnis vom Autor in lateinischen Schriften des frühen und hohen Mittelalters. In Der Autor im Dialog. Beiträge zu Autorität und Autorschaft, hgg. von F.P. Ingold und W. Wunderlich. St. Gallen: 17-31.

Mundt, L. 2008. Die Lehrtätigkeit des Georg Sabinus an der Universität Königsberg. In Die Universität Königsberg in der Frühen Neuzeit, hgg. von H. Marti und M. Komorowski. Köln/Weimar/Wien: 77-115.

Parry, M. 193o. Studies in the Epic Technique of Oral Verse-Making. I. Homer and Homeric Style. Harvard Studies in Classical Philology 41: 73-147.

Petersohn, J. 1963. Fürstenmacht und Ständetum in Preußen während der Regierung Herzog Georg Friedrichs 1578-1603. Würzburg (Marburger Ostforschungen 20).

Schaefer, U.1992. Vokalität. Altenglische Dichtung zwischen Mündlichkeit und Schriftlichkeit. Tübingen (ScriptOralia 39).

Schnell, R. 1984. Prosaauflösung und Geschichtsschreibung im deutschen Spätmittelalter. Zum Entstehen des frühneuhochdeutschen Prosaromans. In Literatur und Laienbildung im Spätmittelalter und in der Reformationszeit, hgg. von L. Grenzmann und K. Stackmann. Stuttgart (Germanistische Symposien Berichtsbände 5): 214-248.

Schucan, L. 1973. Das Nachleben von Basilius Magnus , ad adolescentes. Ein Beitrag zur 
Geschichte des christlichen Humanismus. Genf (Travaux d'Humanisme et Renaissance 133).

Schwitzgebel, B. 1996. Noch nicht genug der Vorrede. Zur Vorrede volkssprachiger Sammlungen von Exempeln, Fabeln, Sprichwörtern und Schwänken des 16. Jahrhunderts. Tübingen (Frühe Neuzeit 28).

Seeck, G.A. 2004. Homer. Eine Einführung. Stuttgart.

Segebrecht, W. 1976. Zur Produktion und Distribution von Casualcarmina. In StadtSchule - Universität - Buchwesen und die deutsche Literatur im 17. Jahrhundert. Vorlagen und Diskussionen eines Barock-Symposions der Deutschen Forschungsgemeinschaft 1974 in Wolfenbüttel, hgg. von A. Schöne. München: 523-535.

Segebrecht, W. 1977. Das Gelegenheitsgedicht. Ein Beitrag zur Geschichte und Poetik der deutschen Lyrik. Stuttgart.

Segebrecht, W. 1997. Gelegenheitsgedicht. $R L W$ 1: 688-691.

Seifert, A. 1984. Der Humanismus an den Artistenfakultäten des katholischen Deutschland. In Humanismus im Bildungswesen des 15. und 16. Jahrhunderts, hgg. von W. Reinhard. Weinheim (Mitteilung der Kommission für Humanismusforschung 12): 135154 .

Von Selle, G. 1956. Geschichte der Albertus-Universität zu Königsberg in Preußen. Zweite Ausgabe. Würzburg.

Sieveke, F.G. 1974. Eloquentia sacra. Zur Predigttheorie des Nicolaus Caussinus S.J. In Rhetorik. Beiträge zu ihrer Geschichte in Deutschland vom 16.-20. Jahrhundert, hgg. von H. Schanze. Frankfurt am Main: 43-68.

Thomae, J. 1580. Oratio D. Basilii de nocentissimo invidiae vicio, carmine heroico translata et illustrata. Königsberg.

Toepfer, R. 2007. Pädagogik, Polemik, Paränese. Die deutsche Rezeption des Basilius Magnus im Humanismus und in der Reformationszeit. Tübingen (Frühe Neuzeit 123).

Toepfer, R. 2008-2009. Mäzenatentum in Zeiten des Medienwechsels. Kaiser Maximilian als Widmungsadressat humanistischer Werke. Jahrbuch der Oswald von Wolkenstein-Gesellschaft 17: 79-92.

Toepfer, R. 20og. Implizite Performativität. Zum medialen Status des Donaueschinger Passionsspiels. $P B B$ 131: 106-113.

Toepfer, R. 2010. Predigtrezeption aus historisch-mediologischer Perspektive. Deutsche Übersetzungen griechischer Kirchenväter im Buchdruck des 16. Jahrhunderts. In Die Predigt im Mittelalter zwischen Mündlichkeit, Bildlichkeit und Schriftlichkeit, hgg. von R. Wetzel und F. Flückiger. Zürich (Medienwandel - Medienwechsel - Medienwissen 13): 37-65.

Töppen, M. 1849. Der lange Königsberger Landtag. Eine Mittheilung aus der älteren preußischen Geschichte. Historisches Taschenbuch N.F. 10: 441-582.

VD 16 (= Bayerische Staatsbibliothek München in Verbindung mit der Herzog August 
Bibliothek Wolfenbüttel (Hg.). 1983-1995. Verzeichnis der im deutschen Sprachraum erschienenen Drucke des XVI. Jahrhunderts. Abt. 1: 22 Bde. Stuttgart.)

Velten, H.R. 2002. Performativität. In Germanistik als Kulturwissenschaft. Eine Einführung in neue Theoriekonzepte, hgg. von C. Benthien u. a. Reinbek: 217-242.

Vessey, M. 1997. English Translations of the Latin Fathers, 1517-1611. In The Reception of the Church Fathers in the West. From Carolingians to the Maurists, hgg. von I. Backus. Leiden u. a.: $775^{-835}$

Vogel, S. 1999. Kulturtransfer in der frühen Neuzeit. Die Vorworte der Lyoner Drucke des 16. Jahrhunderts. Tübingen (Spätmittelalter und Reformation Neue Reihe 12).

Walter, A.E. 2004. Das Schicksal der Königsberger Archive und Bibliotheken - Eine Zwischenbilanz. In Königsberger Buch- und Bibliotheksgeschichte, hgg. von A.E. Walter. Köln/Weimar/Wien (Aus Archiven, Bibliotheken und Museen Mittel- und Osteuropas 1): 1-68.

Walter, A.E. 2005. Die Bibliothek der Staatlichen Immanuel Kant-Universität Kaliningrad und die ehemalige Königsberger Staats- und Universitätsbibliothek einschließlich der Wallenrodtschen Sammlung. Eine bibliotheksgeschichtliche Darstellung und ein Überblick über die Bestände vom Mittelalter bis in die Gegenwart. In Handbuch des personalen Gelegenheitsschriftums in europäischen Bibliotheken und Archiven, hgg. von S. Beckmann, K. Garber und A.E. Walter, Bd.16: Königsberg - Bibliothek der Russischen Staatlichen Immanuel Kant-Universität. Hildesheim/Zürich/New York: $21-67$.

Wetzel, R., und F. Flückiger (Hg.) 2010. Die Predigt im Mittelalter zwischen Mündlichkeit, Bildlichkeit und Schriftlichkeit. Zürich (Medienwandel - Medienwechsel - Medienwissen 13).

Zumthor, P. 1987. La lettre et la voix de la ,littérature medieval. Paris. 